\title{
OPTIMUM TRANSONIC AIRFOILS BASED ON THE EULER EQUATIONS
}

\author{
Angelo Iollo ${ }^{1}$ \\ Dipartimento di Ingegneria Aeronautica e Spaziale \\ Politecnico di Torino \\ 10129 Torino, Italy \\ Manuel D. Salas \\ Institute for Computer Applications in Science and Engineering \\ NASA Langley Research Center \\ Hampton, VA 23681-0001
}

\begin{abstract}
We solve the problem of determining airfoils that approximate, in a least square sense, given surface pressure distributions in transonic flight regimes. The flow is modeled by means of the Euler equations and the solution procedure is an adjointbased minimization algorithm that makes use of the inverse Theodorsen transform in order to parameterize the airfoil. Fast convergence to the optimal solution is obtained by means of the pseudo-time method. Results are obtained using three different pressure distributions for several free stream conditions. The airfoils obtained have given a trailing edge angle.
\end{abstract}

\footnotetext{
${ }^{1}$ This research was supported in part under NASA contract no. NAS1-19480 while the first author was in residence at the Institute for Computer Applications in Science and Engineering, NASA Langley Research Center, Hampton, VA 23681-0001.
} 



\section{Introduction}

State of the art computational techniques allow the analysis of complex two-dimensional or three-dimensional configurations of aerodynamic interest, using models of increasing complexity. In the design of aerodynamic components however, simpler physical models have been used, in order to obtain analytical solutions or tractable computational problems. In some cases, the use of simpler models does not impair the validity of the results obtained. For example, transonic shock-free airfoils were designed using the full potential equation written on the hodograph plane: since shock-free solutions were sought, it was not necessary to use the Euler equations.

This simplification is generally not valid when shocks, even weak shocks, are present in the flow field. In fact, although entropy production by weak shocks could be small, at shock points of high curvature, entropy gradients could be high as pointed out by Nieuwland \& Spee [18]. Another reason to avoid the potential approximation in the presence of shocks is the possibility of multiple solutions as shown by Steinhoff \& Jameson [24]. Therefore, since shock-free solutions are at least unlikely when designing airfoils in the presence of constraints of various kinds, the use of the Euler equations becomes necessary.

Much effort in transonic aerodynamics has been devoted to the design of shock-free airfoils, even though according to an early theoretical result due to Morawetz [16], shock-free solutions of the full potential equations are isolated points. However, subsequent experimental investigation conducted by Pearcy [20], and by Nieuwland \& Spee [17] showed that shock-free flows around airfoils with no or weak shocks can be experimentally realized and 
are stable with respect to unsteady disturbances or variations of $M_{\infty}$. The design methods employed at that time were based on the transformation of a known solution in the hodograph plane to the physical plane, and iterating on the input hodograph solution based on to the result obtained in the physical plane. For a review of these methods see the article by Boerstoel [4]. A common drawback of these approaches is that they have very little control on the resulting pressure distribution over the airfoil. Volpe [27] tried to overcome this problem by taking pressure distribution as an input design requirement. However, for the well posedness of the problem, special restrictions had to be applied on the possible choices. In addition, the flows obtained with such airfoils showed secondary shocks embedded in the flow field, that caused losses even at design conditions and boundary layer separation at off-design conditions, as pointed out by Labrujère \& Slooff [15].

More recently, advances in design methodology have come about through shape optimization. Shape optimization is based on the minimization of an objective function, defined on the boundary, with respect to the variation of the boundary itself. This approach allows for example multi-point design and the easy introduction of design constraints, in addition to control of the pressure distribution on the body. The first attempts of Pironneau [21][22] and of Glowinski \& Pironneau[7] to solve such problems, were based on control theory. With this approach the minimum of the functional is determined with a descent algorithm based on the gradient of the functional with respect to the controls. The gradient of the functional is determined solving the adjoint of the equations governing the flow. No much progress was made at the time because no code for the computation of the flow field and of 
the adjoint equations was available. Recently this method was reintroduced by Jameson [13] and computationally demonstrated its inherent vantages. In fact, with other minimization strategies for wing design, such as that first proposed by Hicks \& Henne [9], the gradient of the functional is computed by finite differences, perturbing the controls one by one. This requires a complete flow-field solution for each gradient component to be computed. Even though optimal designs have been obtained with this method for the potential equations, see Reuther, van Dam \& Hicks [23], the method becomes too computationally expensive for the Euler and Navier-Stokes equations. In contrast, with the method first proposed by Pironneau [21], it is necessary to compute the flow field only once for each gradient evaluation. This has led to several applications for both internal and external flows governed by Euler equations - see Beux \& Dervieux [2], Jameson \& Reuther [14], Iollo \& Salas [11] - for Navier-Stokes equations, see Cabuk \& Modi [5]. In the latter work, however, the cost of computations was so high that they could only afford to compute about 10 flow-field solutions on a relatively coarse grid.

A further decrease in the computational effort is possible by applying the theory proposed by Ta'asan [25], namely the pseudo-time method, which is based on the following observation. Gradient-based methods (including adjoint formulations) can be viewed as marching along the intersection of the hypersurfaces representing the solutions of the flow-field and of the adjoint equations. This is an expensive process since each step requires the solution of two systems of PDEs. The idea of the pseudo-time method is to perform the marching while satisfying the optimality conditions on the boundary, but without satisfying the 
flow-field and adjoint equations in the interior domain. The cost of such an iteration per step is significantly smaller than that of gradient-based methods. Its convergence has been shown by Ta'asan to be independent of the number of design variables. This method was applied by Iollo, Kuruvila \& Ta'asan [10] to a few model problems governed by the Euler equations, which require the solution of the optimization problem in a small vicinity of the boundary. The results were compatible with the theoretical predictions.

In the present paper we use the above method for transonic airfoil design. In particular we are concerned with the problem of finding families of wing sections that have approximately the same pressure distribution at different flight conditions. An example of such airfoils was given by Chin [6]. In this paper we show a few families corresponding to different pressure distributions, with prescribed trailing edge angle. Such families are designed by means of the inverse Theodorsen transform, that allows the optimization with an increasing number of harmonics defining the airfoil geometry. Compared to previous work, we are able

to design for given pressure distribution using the Euler equations, even in the presence of flows with sharply captured shocks. The total computational cost to reach an optimum is of the order of the cost of a few flow-field analyses, allowing a cheap mapping of performance with several target pressure distributions.

\section{Statement of the Problem}

Consider a plane transonic flow around an airfoil as in fig. 1. Let the upstream Mach number be $M_{\infty}$ and take the unperturbed stream pressure and density as reference. Disregarding 


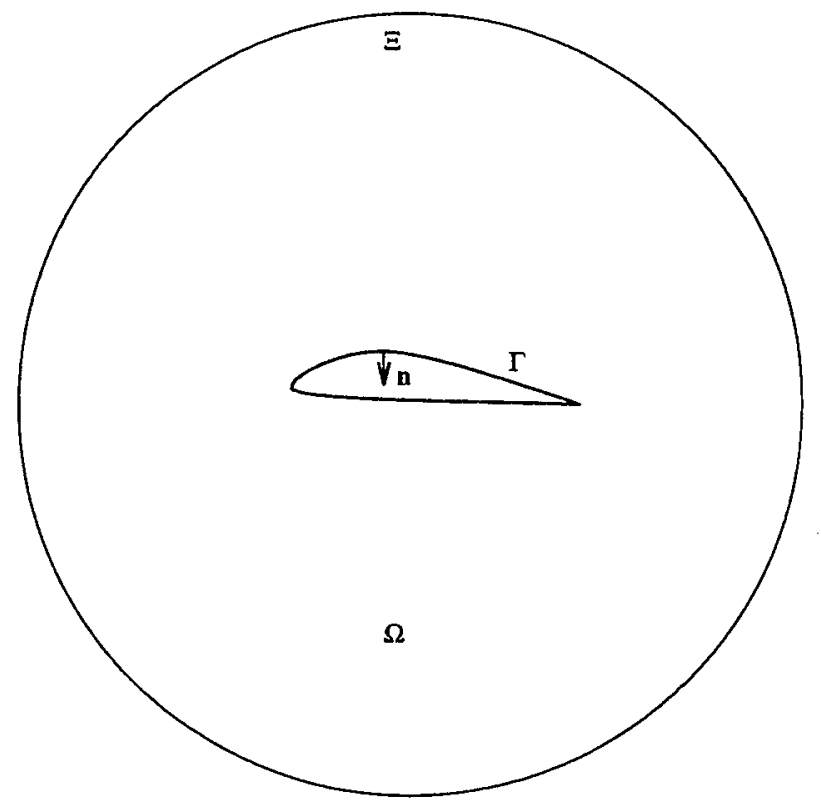

Figure 1: Physical plane. The portion of the plane included in $\Xi$, and external to $\Gamma$ is denoted by $\Omega$.

viscous effects, the flow is governed by the Euler equations written in the following form

$$
U_{t}+F_{x}+G_{y}=0
$$

where

$$
U=\left[\begin{array}{c}
\rho \\
\rho u \\
\rho v \\
\rho e
\end{array}\right] \quad F=\left[\begin{array}{c}
\rho u \\
p+\rho u^{2} \\
\rho u v \\
u(\rho e+p)
\end{array}\right] G=\left[\begin{array}{c}
\rho v \\
\rho u v \\
p+\rho v^{2} \\
v(\rho e+p)
\end{array}\right]
$$

Here $u, v, \rho, e$ and $p$ are the velocity components, density, specific total energy and pressure respectively. Assuming a perfect gas law, we also have $p=\kappa \rho\left(2 e-u^{2}-v^{2}\right)$ with $\kappa=$ $(\gamma-1) / 2$ and $\gamma$ the ratio of specific heats. The Euler equations define the state of the 


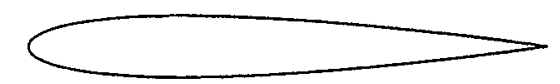

a)

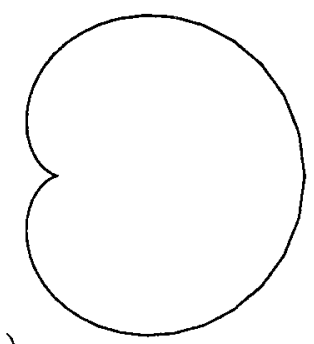

b)

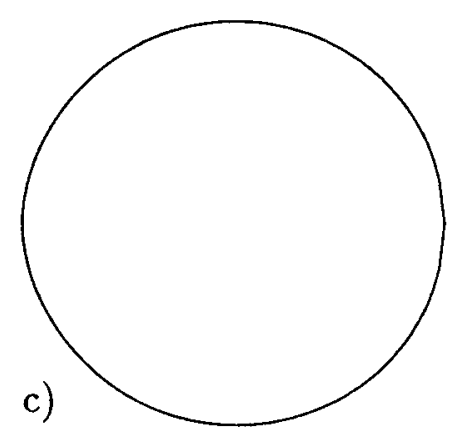

Figure 2: a) $z$ plane; b) $w$ plane; c) $w^{\prime \prime}$ plane.

system, and therefore are named state equations.

We assume $M_{\infty}<1$ and that the external boundary $\Xi$ is far enough so that the flow is unperturbed there. If the flow is incoming with respect to the normal to $\Xi$, total pressure, entropy and the ratio $v / u$ are assigned, whereas if the flow is outgoing static pressure is prescribed. The boundary condition on the airfoil is $\rho \mathbf{V} \cdot \mathbf{n}=0$, where $\mathbf{n}$ is the normal to $\Gamma$ oriented as in fig. 1 and $\mathbf{V}=(u, v)$.

Consider a real valued functional $\mathcal{E}(\Gamma, X(\Gamma))$ where $X(\Gamma)$ is a solution of the Euler equations with boundary conditions. The optimization problem consists of minimizing the functional $\mathcal{E}$ over all the admissible shapes of the boundary $\Gamma$.

The functional $\mathcal{E}$ is chosen according to some design criteria. Although the formulation we propose is general, here we will limit ourself to the case in which

$$
\mathcal{E}(\Gamma)=\frac{1}{2} \oint_{\Gamma}\left[p(\Gamma)-p^{*}\right]^{2} d s
$$

where $p^{*}$ is a given pressure distribution and $s$ is the curvilinear coordinate on $\Gamma$. The solution of such problem will allow the design of minimum-wave-drag profiles in transonic 


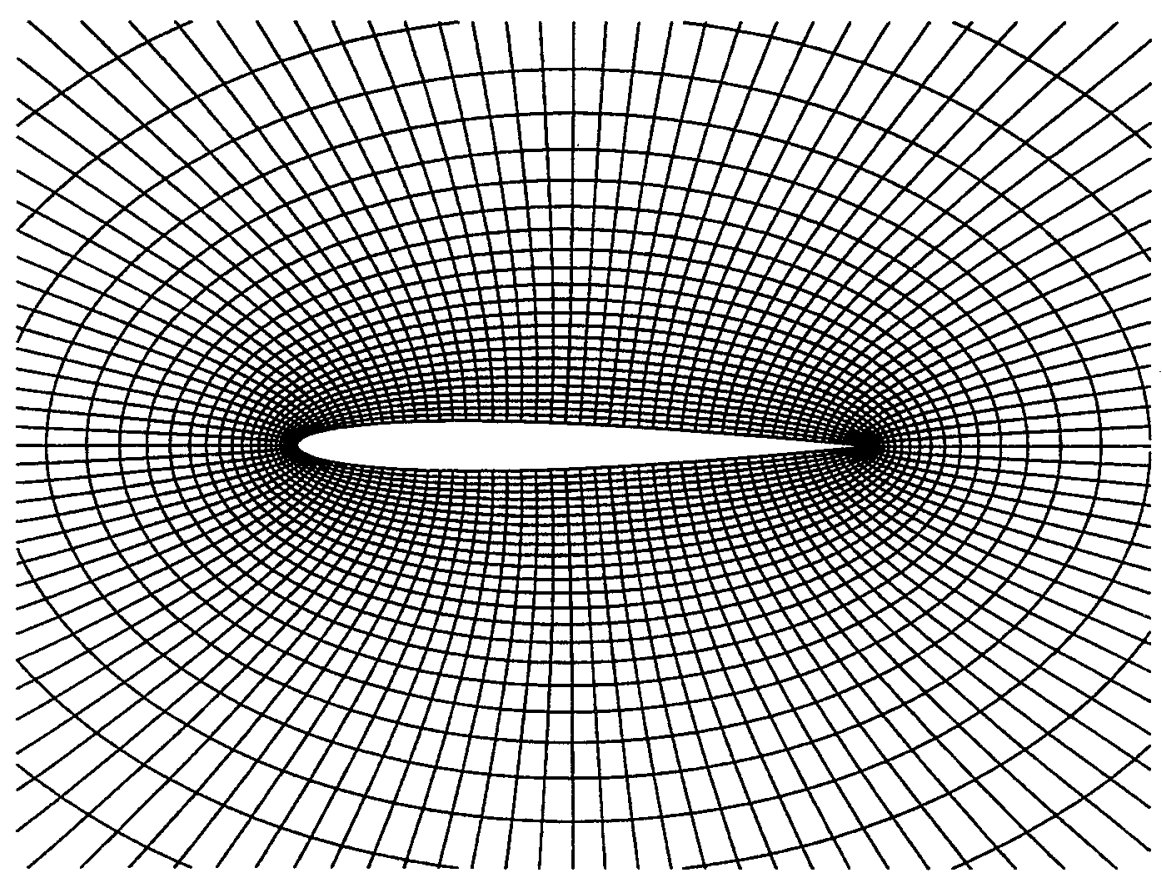

Figure 3: Computational mesh.

flows. Constraints on aerodynamic characteristics of the airfoil such as pitching moment or lift coefficient can be added to $\mathcal{E}$ in the form of quadratic penalty functions.

\section{Analysis}

In this section we will derive the necessary conditions for the minimum of $\mathcal{E}(\Gamma)$ subject to the Euler equations. The derivation is similar to that presented for internal flows by Iollo $\&$ Salas [11]. Consider the augmented functional

$$
\mathcal{L}(U, \Gamma, \Lambda, \mu)=\mathcal{E}+\int_{\Omega}{ }^{t} \Lambda\left(A U_{x}+B U_{y}\right) d \Omega+\int_{\Gamma} \mu \rho \mathbf{V} \cdot \mathbf{n} d s
$$

where $A=\partial F / \partial U$ and $B=\partial G / \partial U$. The vector $\Lambda(x, y)={ }^{t}\left(\lambda_{1}, \lambda_{2}, \lambda_{3}, \lambda_{4}\right)$ and the scalar $\mu(s)$ are the continuous equivalents of the Lagrange multipliers. We will show that the 
unconstrained minimum of eq. 3 with respect to $U, \Gamma, \Lambda$ and $\mu$ is the same as that of $\mathcal{E}(\Gamma)$.

Suppose each point of the airfoil can be displaced a length $\varepsilon \tilde{n}(s)$ in the direction of the normal $\mathrm{n}$ to the profile in a way that will be described in the next section. Corresponding to this change, $U(x, y)$ is increased by a function $\varepsilon \widetilde{U}(x, y) ; \Lambda(x, y)$ by $\varepsilon \widetilde{\Lambda}(x, y)$ and $\mu(s)$ by $\varepsilon \tilde{\mu}(s)$. Calculating the variation of the functional $\mathcal{L}$ with respect to the variation of $U, \Lambda$, $\mu$ and $\Gamma$ respectively, and disregarding higher order terms we obtain

$$
\begin{aligned}
\delta \mathcal{L}_{U} & =\oint_{\Gamma} \frac{\partial p}{\partial U}\left(p(\Gamma)-p^{*}\right) \tilde{U} d s+\int_{\Gamma}{ }^{t} \Lambda\left(A n_{x}+B n_{y}\right) \tilde{U} d s+ \\
& -\int_{\Omega}\left({ }^{t} \Lambda_{x} A+{ }^{t} \Lambda_{y} B\right) \tilde{U} d \Omega+\int_{\Gamma} \mu \mathbf{n} \cdot \frac{\partial \rho \mathbf{V}}{\partial U} \tilde{U} d s
\end{aligned}
$$

where

$$
\frac{\partial p}{\partial U}=2 k\left(\frac{u^{2}+v^{2}}{2},-u,-v, 1\right) \text { and } \frac{\partial \rho \mathbf{V}}{\partial U}=\left(\begin{array}{cccc}
0 & 1 & 0 & 0 \\
0 & 0 & 1 & 0
\end{array}\right)
$$

and

$$
\begin{aligned}
\delta \mathcal{L}_{\Lambda} & =\int_{\Omega}{ }^{t} \tilde{\Lambda}\left(A U_{x}+B U_{y}\right) d \Omega \\
\delta \mathcal{L}_{\mu} & =\oint_{\Gamma} \tilde{\mu} \rho \mathbf{V} \cdot \mathbf{n} d s \\
\delta \mathcal{L}_{\Gamma} & =\oint_{\Gamma} \frac{\partial p}{\partial n}\left(p(\Gamma)-p^{*}\right) \tilde{n} d s+\int_{\Gamma}{ }^{t} \Lambda\left(A U_{x}+B U_{y}\right) \tilde{n} d s+ \\
& +\int_{\Gamma} \mu \frac{\partial(\rho \mathbf{V})}{\partial n} \cdot \mathbf{n} \tilde{n} d s-\int_{\Gamma} \mu \rho \mathbf{V} \cdot \tilde{\mathbf{n}} d s
\end{aligned}
$$

where $\tilde{\mathbf{n}}$ is the variation of the normal to the airfoil boundary $\Gamma$.

At the minimum of the functional, for all the possible choices of the functions $\tilde{U}, \tilde{\Lambda}, \tilde{\mu}$ and $\tilde{n}$, the following must be true

$$
\delta \mathcal{L}_{U}=\delta \mathcal{L}_{\Lambda}=\delta \mathcal{L}_{\mu}=\delta \mathcal{L}_{\Gamma}=0
$$


Therefore, we have

$$
\delta \mathcal{L}_{\Lambda}=0 \Leftrightarrow A U_{x}+B U_{y}=0 \text { on } \Omega
$$

and

$$
\delta \mathcal{L}_{\mu}=0 \Leftrightarrow \rho \mathbf{V} \cdot \mathbf{n}=0 \text { on } \Gamma
$$

which are the Euler equations and boundary conditions. Furthermore we obtain the following adjoint or costate equations

$$
\delta \mathcal{L}_{U}=0 \Leftrightarrow{ }^{t} A \Lambda_{x}+{ }^{t} B \Lambda_{y}=0 \text { on } \Omega
$$

On the airfoil, eqs. 9 become

$$
\frac{\partial p}{\partial U}\left(p(\Gamma)-p^{*}\right)+{ }^{t} \Lambda\left(A n_{x}+B n_{y}\right)+\mu \mathbf{n} \frac{\partial \rho \mathbf{V}}{\partial U}=0
$$

where

$$
\mu=-\left[\lambda_{1}+u \lambda_{2}+v \lambda_{3}+\left(\gamma e-k V^{2}\right) \lambda_{4}\right]
$$

From eq. 10 the boundary condition for $\Lambda$ on $\Gamma$ are found. Given $U$, the set of costate eqs.(9-11) determine $\Lambda$ in $\Omega$ and $\mu$ on $\Gamma$ uniquely.

It is seen that if we could solve for $\tilde{n}$ from the system of eqs. 8 , then we would also determine the minimum of $\mathcal{E}$. Unfortunately, eqs. 8 cannot be easily solved for $\tilde{n}$. Nevertheless, using an optimization algorithm it will be possible to determine the shape of $\Gamma$ that minimizes $\mathcal{L}$ and $\mathcal{E}$. In fact, knowing $U$ and $\Lambda$ for a given shape of the airfoil, we can calculate from eq. (7) how to choose $\tilde{n}$ so that $\delta \mathcal{L}<0$, and iterate until the minimum is reached. Two algorithms of this kind will be discussed in Section 5. 
In case of shocks occuring in the flow field, we split the domain of integration by means of a curve $\Upsilon$ that coincides with the shocks where they exist. Then we follow the same derivation presented so far on each of the sub-domains, regarding $\Upsilon$ as a boundary; the situation is similar to that which occurs for internal two-dimensional flows. The resulting extra condition for $\Lambda$ on the shock is $\Lambda=0$. It should be noted that if the shocks are not treated properly, the problem of solving the costate equations with boundary conditions is not well-posed, as shown by Iollo, Salas \& Ta'asan [12].

\section{Airfoil Design by Inverse Theodorsen Transform}

By means of a conformal mapping, it is possible to transform a large class of shapes of aerodynamic interest to the unit circle. In particular, using the Theodorsen transform it is possible to transform any given profile to the unit circle. As an example, we will transform the NACA 0012 profile of fig. 2a. First the profile is represented in the complex plane $z$, with the trailing edge corresponding to the point $(1,0)$. Then the plane $z$ is transformed into the plane $w$ (fig. $2 \mathrm{~b}$ ) using the relation

$$
w=\frac{z-1}{z+1}
$$

The angle in the origin is eliminated by the transformation

$$
w^{\prime}=w^{\tau}
$$

where

$$
\tau=\frac{\pi}{2 \pi-\alpha}
$$


where $\alpha$ is the angle at the trailing edge. Finally, the plane $w^{\prime}$ is transformed into the quasi-circle of fig. $2 c$ by

$$
w^{\prime \prime}=\frac{1-w^{\prime}}{w^{\prime}+1}
$$

If we denote by $\xi$ the unit circle plane, the transform is completed by

$$
w^{\prime \prime}=\xi e^{\sum_{n=0}^{N}\left(a_{n}+i b_{n}\right) \xi^{-n}}
$$

where the coefficients $a_{n}$ and $b_{n}$ are usually determined by means of the Fast Fourier Transform.

In the design of an airfoil, we proceed in the opposite way. Starting from the unit circle plane, the coefficients $a_{n}$ and $b_{n}$ are given so that an airfoil is obtained on the $z$ plane. Let us define $\xi=r e^{i \varphi}$ and $w^{\prime \prime}=\rho e^{i \theta}$. In order to design an airfoil with a given trailing edge angle, it is necessary that the figure resulting on the plane $w^{\prime \prime}$ passes through the point $(1,0)$. In addition it is convenient that for $\varphi=0$ we have $\theta=0$. It is easily seen that for $\varphi=0$, the radius $r$ is not affected by the value of $b_{0}$, while $\theta=\sum_{n=0}^{N} b_{n}$. Hence, taking $b_{0}=-\sum_{n=1}^{N} b_{n}$, we have $\varphi=0$ for $\theta=0$. Finally, since the value of $\theta$ is not affected by the coefficient $a_{0}$, we can take $a_{0}=-\sum_{n=1}^{N} a_{n}$ in order to have $\rho=1$ for $\varphi=0$.

The coefficients must be evaluated accurately to obtain reasonable shapes in the $z$ plane. For example, in the case of non lifting profiles $b_{n}=0$ for every $n$. On the other hand, the summation $\sum_{n=0}^{N} n b_{n}$ controls the camber, as can be seen studying the transform in the vicinity of $\varphi=0$.

Given a set of coefficients $a_{n}$ and $b_{n}$ we are now interested in calculating the variation of the tangent and of the normal when we increment one of the coefficients by a small quantity, 
say $\delta a_{i}$. Given two points $z$ and $z^{\prime}$ on the airfoil, the tangent to the profile is defined by

$$
\lim _{z^{\prime} \rightarrow z} \frac{z^{\prime}-z}{\left|z^{\prime}-z\right|}
$$

Let us consider the corresponding points on the unit circle, respectively $\xi^{\prime}$ and $\xi$, we have

$$
\lim _{\xi^{\prime} \rightarrow \xi} \frac{\xi^{\prime}-\xi}{\left|\xi^{\prime}-\xi\right|}=e^{i(\varphi+\pi / 2)}
$$

Therefore we obtain

$$
\lim _{z^{\prime} \rightarrow z} \frac{z^{\prime}-z}{\left|z^{\prime}-z\right|}=\left|\frac{d H}{d \xi}\right| e^{i(\varphi+\pi / 2)}
$$

where $H$ is the transformation from $\xi$ to $z$. For example, the variation of the normal with respect to $a_{i}$ is given by

$$
\tilde{\mathbf{n}}=e^{i \varphi} \frac{\partial}{\partial a_{i}}\left(\left|\frac{d H}{d \xi}\right|\right) \delta a_{i}
$$

Often airfoils are subject to geometric constraints such as fixed volume, maximum or minimum thickness, or given trailing edge angle. These constraints can be easily included in the mapping formulation. For example, if $\tau$ is kept constant, then the trailing edge angle is constant.

On the other hand, the volume included by an airfoil is

$$
V=\Re(\oint \bar{z} d z)
$$

and hence it is possible to compute the variation of the volume with respect to the coefficients of the transformation. Therefore, when the volume is kept fixed, the equation

$$
\sum_{n=0}^{N} \frac{\partial V}{\partial a_{n}} \delta a_{n}+\sum_{n=0}^{N} \frac{\partial V}{\partial b_{n}} \delta b_{n}=0
$$


represents the additional constraint in the minimization. In particular, we can take for example

$$
\delta a_{i}=-\frac{\sum_{n=0, n \neq i}^{N}\left(\partial V / \partial a_{n}\right) \delta a_{n}+\sum_{n=0}^{N}\left(\partial V / \partial b_{n}\right) \delta b_{n}}{\partial V / \partial a_{i}}
$$

and substitute $\delta a_{i}$ in the expression for the descent direction, in order to obtain a constrained minimization.

Taking the coefficients $a_{n}$ and $b_{n}$ to be the controls, we were able to perform a minimization with an increasing number of controls involved. In fact, in the first optimization iteration, the rough shape of the airfoil must be defined, therefore it is needless to work with many Fourier coefficients at this point. As the rate of convergence to the minimum decreases, higher harmonics are added until the convergence rate increases and the functional is minimized.

\section{Numerical Solution}

In this section we will discuss the numerical solution of the flow-field equations and of the adjoint equations. In addition, two solution strategies for the minimization problem of Section 2 will be presented.

The Euler equations are solved by means of an explicit, finite-volume scheme based on the flux-difference formulation proposed by Pandolf [19], and the steady solution is obtained as an asymptotic limit of a time evolution. The conservative variables $U$ are computed at the cell centers, and the fluxes $F$ and $G$ are evaluated at the cell interfaces with an approximate Riemann solver. Second-order accuracy is achieved using a method in the spirit of the 


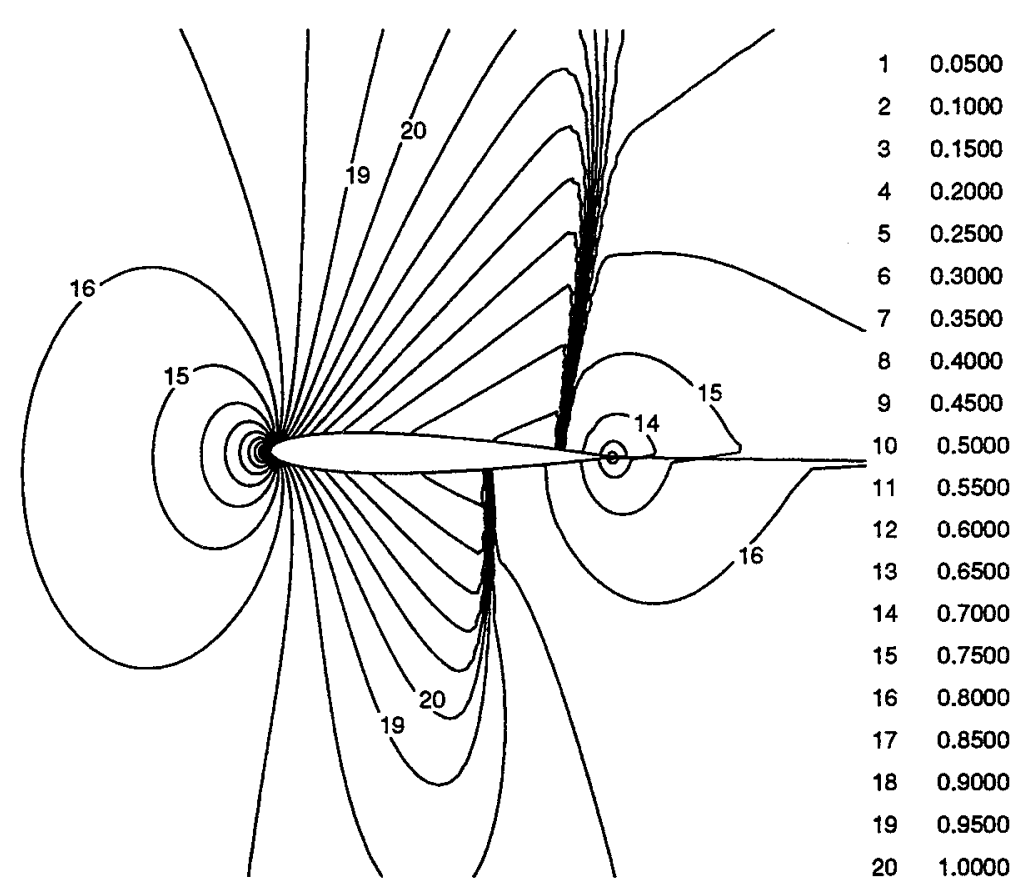

Figure 4: Iso-Mach lines for a NACA 0012 at 1 degree of incidence and $M_{\infty}=0.85$. $C_{L}=0.334, C_{D}=0.0568$

essentially non-oscillatory scheme of Harten, Engquist \& Chakravarthy [8]. With such an approach the flow-field values at the cell interfaces, used as initial conditions for the Riemann problem, are reconstructed by means of a linear interpolation. The occurrence of spurious oscillations is prevented using a minmod limiter. The amplitude of the integration step is chosen in accordance with the Courant-Friedrichs-Lewy (CFL) condition.

Compared to other solvers, this one allows a sharper capturing of shocks and contact discontinuities, so that wave-drag computations are more reliable. It should be emphasized that in other works, such that of Jameson \& Reuther [14], no special treatment of the costate equations is made at the shock. This is allowed in their formulation because shocks are smeared over several grid points due to artificial viscosity. However, because of the 


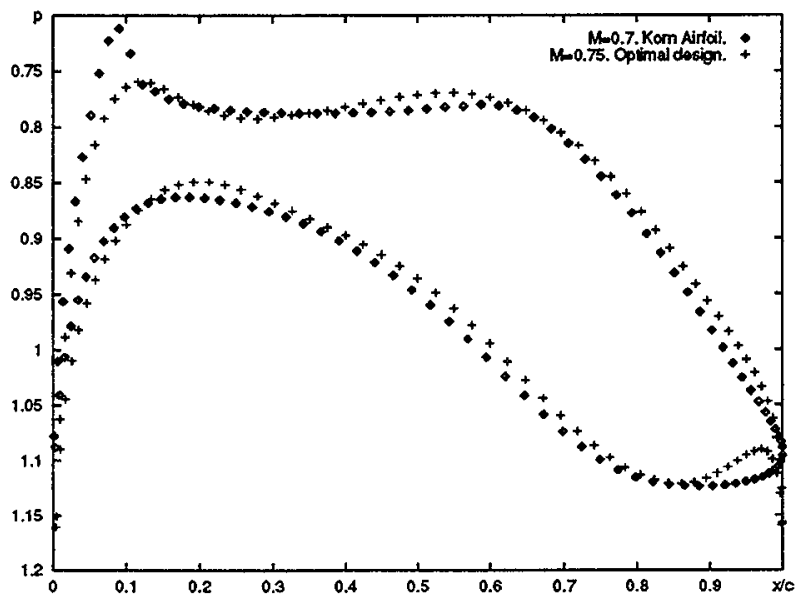

Figure 5: Pressure versus chord fraction for Korn airfoil and optimal airfoil at $M_{\infty}=0.75$. The unperturbed flow static pressure is taken as reference.

smearing of the shock, the computed gradient might not be reliable close to the minimum, causing a drastic reduction of the convergence rate.

The costate equations have no conservative form, and the numerical solution is obtained with a finite-difference scheme. We introduce a set of curvilinear coordinates $\varphi(x, y)$ and $\psi(x, y)$. The costate equations are then rewritten as follows

$$
{ }^{t} \mathcal{A} \Lambda_{\varphi}+{ }^{t} \mathcal{B} \Lambda_{\psi}=0
$$

where $\mathcal{A}=A \varphi_{x}+B \varphi_{y}$ and $\mathcal{B}=A \psi_{x}+B \psi_{y}$. The transformations $\varphi$ and $\psi$ are defined as $\left(x_{l}, y_{m}\right) \stackrel{\varphi}{\rightarrow} l$ and $\left(x_{l}, y_{m}\right) \stackrel{\psi}{\rightarrow} m$, where $l$ and $m$ are the indices that characterize each grid point. The above equations are linear and as such are their boundary conditions, see Iollo \& Salas [11]. Suppose that locally we separate the variables through the following approximation:

$$
\Lambda(\varphi, \psi, t)=\Lambda^{\prime}(\varphi, t)+\Lambda^{\prime \prime}(\psi, t)
$$




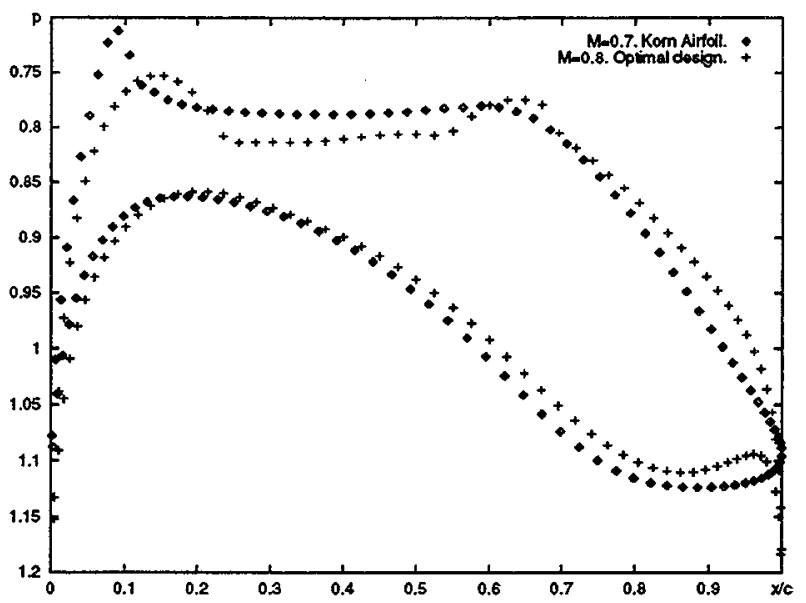

Figure 6: Pressure versus chord fraction for Korn airfoil and optimal airfoil at $M_{\infty}=0.8$. The unperturbed flow static pressure is taken as reference.

We are left with two one-dimensional subproblems that are solved as the asymptotic limit of a time-dependent technique, as explained by Iollo \& Salas [11].

The computational grid is the same for Euler and adjoint equations and is obtained by means of the transformation that we use to generate the profile. All the computations have been performed on an O-grid with 120 tangential and 30 radial points, as partially shown in fig. 3. The outer boundary is at 10 chords from the airfoil.

In Section 3 we have introduced the basic idea for the minimization of the functional $\mathcal{L}$. From eq. 7 and eq. 19, once the solution of Euler and adjoint equations is computed for the present geometry, it is possible to calculate the gradient of $\mathcal{L}$ with respect to the coefficients $a_{n}$ and $b_{n}$. In particular, disregarding higher order terms, we have

$$
\delta \mathcal{L}=\sum_{n=1}^{N}\left[\frac{\partial \mathcal{L}}{\partial a_{n}} \delta a_{n}+\frac{\partial \mathcal{L}}{\partial b_{n}} \delta b_{n}\right]
$$



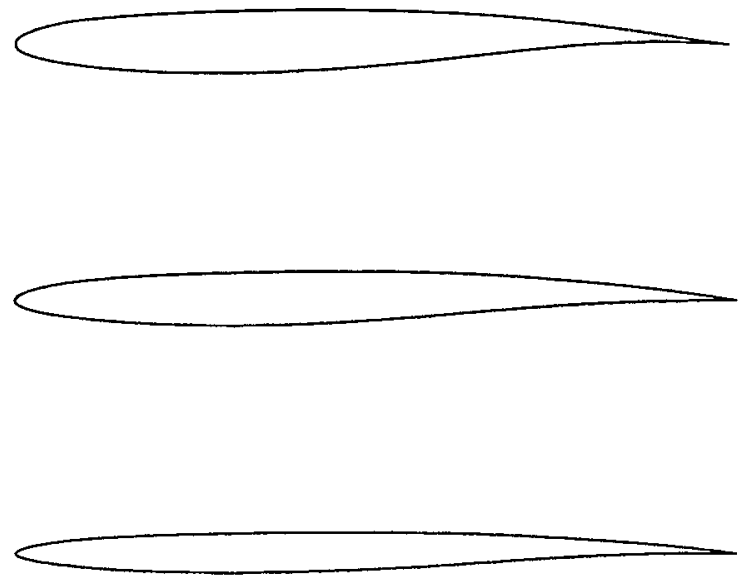

Figure 7: Korn airfoil and optimal designs at $M_{\infty}=0.75$ and $M_{\infty}=0.8$.

and taking

$$
\delta a_{n}=-v \partial \mathcal{L} / \partial a_{n}
$$

and

$$
\delta b_{n}=-v \partial \mathcal{L} / \partial b_{n}
$$

it is $\delta \mathcal{L}<0$ if $v$ is small enough. A first minimization algorithm is therefore:

1. start with a set of $a_{n}$ and $b_{n}$;

2. enforce $\delta \mathcal{L}_{\Lambda}=0$ and $\delta \mathcal{L}_{\mu}=0$ by finding a $U$ that satisfies the steady state Euler equations and boundary conditions;

3. enforce $\delta \mathcal{L}_{U}=0$ by finding a $\Lambda$ that satisfies the costate equations and boundary conditions; 


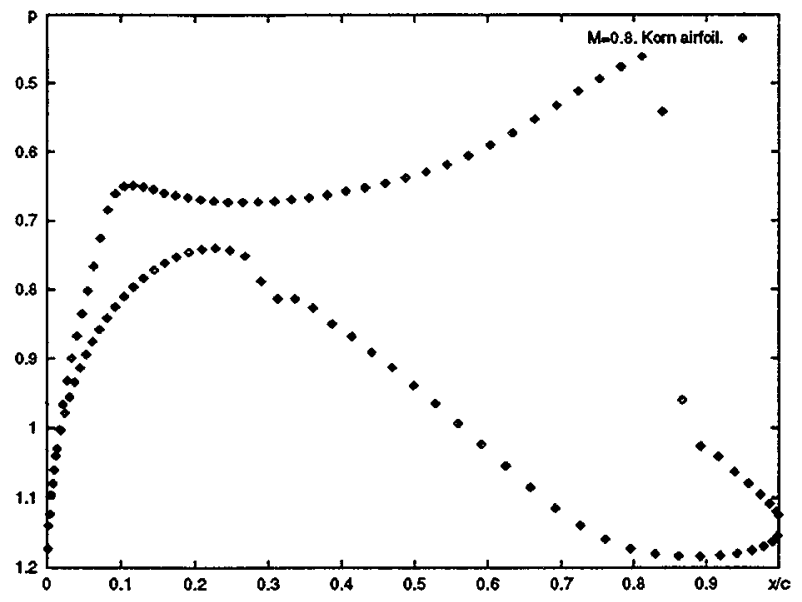

Figure 8: Pressure distribution over a Korn airfoil at $M_{\infty}=0.8$.

4. calculate $\partial \mathcal{L} / \partial a_{n}$ and $\partial \mathcal{L} / \partial b_{n}$ for $n=1 \ldots N$. If these partial derivatives are all 0 we have found the minimum, otherwise

5. update $a_{n}$ and $b_{n}$ for $n=1 \ldots N$ with $\delta a_{n}$ and $\delta b_{n}$ as in eqs. 26 and 27;

6. restart from 2 .

This algorithm is known as steepest descent. In other approaches, like in the quasi-Newton methods, the local curvature of the functional is taken into account in order to compute an optimal descent step $v$ and increase the convergence rate to the minimum.

The adjoint-equations approach reduces the cost of computing the gradient; the cost is the same regardless of the number of controls. However, the number of flow-field evaluations to reach the minimum is very high, and grows more than linearly when the number of controls are increased, as shown by Beux \& Dervieux [3]. Considering flows governed by the NavierStokes equations, as done by Cabuk \& Modi [5], it is seen that only a few minimization steps with a relatively coarse grid are possible, due to the excessive computational times 
required.

Ta'asan [25] proposed the pseudo-time method as a more efficient way of solving the optimization problem. The main observation is the following. The solution of the optimization problem lies on the intersection of the hypersurfaces where the state, costate and optimality conditions are satisfied. Gradient based methods (including adjoint formulations) can be viewed as marching along the intersection of the state and costate hypersurfaces. This is an expensive process since at each step taken along the intersection requires the solution of two systems of PDEs. The idea of the pseudo-time method is to perform the marching on the optimality conditions hypersurface while relaxing the requirement to lie on the state and costate hypersurfaces. Note that the hypersurface representing the optimality conditions is one dimension less that of the state and costate equations. Therefore, the cost of such an iteration per step is significantly smaller than that of gradient based methods. Its convergence has been shown by Ta'asan [25] to be independent of the number of design variables.

In some cases the optimality-conditions equations can be solved for the design variables and a simple implementation of the above idea exists. In other cases the optimalityconditions equations, viewed as an equation for the design variables keeping the state and costate fixed, may be singular and a more involved implementation is required. This is the case for the problem considered here. In such cases it is necessary to solve for the design variables together with the state and costate variables in a small neighborhood of the airfoil boundary, as shown by Iollo, Kuruvila \& Ta'asan [10]. The resulting algorithm is as follows: 
1. start with a tentative set of $a_{n}$ and $b_{n}$;

2. march the Euler equations, in time, a few steps on the entire field;

3. march the adjoint equations, in time, a few steps on the entire field;

4. solve in a small neighborhood of the airfoil boundary $\Gamma$ the Euler equations and satisfy the boundary conditions, likewise do the same with the adjoint equations [10]; then compute the gradient of the functional;

5. if the gradient is null, restart from step 2, repeating steps 3 to 5 until the state and costate equations are converged on the entire field. Otherwise update $a_{n}$ and $b_{n}$ with $\delta a_{n}$ and $\delta b_{n}$ as in eqs. 26 and 27 , go to 4 .

When this algorithm is used in conjunction with the inverse Theodorsen transform, convergence is obtained while increasing the number of coefficients used. Usually we started with $N=2$ and increased $N$ to the point where there is no appreciable decrease in the functional, usually for $N=6$. All of the results presented in the next section are obtained by means of the pseudo-time method. The gradient is considered zero when it has decreased two orders of magnitude, while the functional $\mathcal{E}$ decreases differently according to the case considered. The computational cost of each optimization was about 5 times the cost of a single analysis of the flow field. 


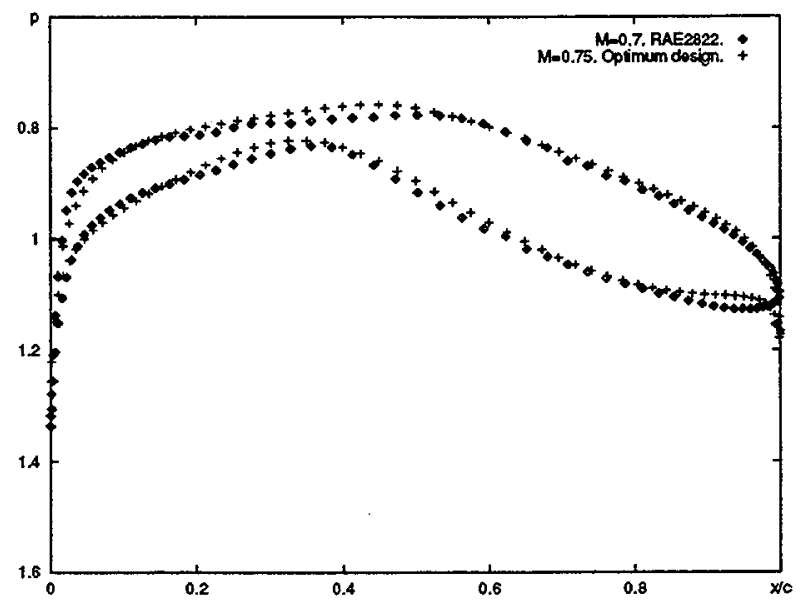

Figure 9: Pressure versus chord fraction for RAE2822 airfoil and optimal airfoil at $M_{\infty}=$ 0.75. The unperturbed flow static pressure is taken as reference.

\section{Results and Discussion}

The analysis code was validated against known test cases. In particular we considered those contained in AGARD [1]. For example, we show the results for test case 02: a NACA 0012 at 1 degree of incidence and $M_{\infty}=0.85$. The grid used had 240 tangential and 60 radial points. The lift coefficient reported for this test case varies from 0.330 to 0.3889 , the one that we find is 0.334 . The drag coefficients reported range from 0.0464 to 0.0590 ; we have 0.0568 . The iso-Mach lines of fig. 4 compare well with the benchmark of fig. 6.19 in AGARD [1].

In order to design an airfoil of some interest, it is necessary that it has some desirable aerodynamic characteristics, such as minimum drag, high lift, or given pitching moment. In addition some structural constraints, for example on the volume or on the trailing edge angle, must be satisfied. In principle, it would be possible to minimize wave drag for given 


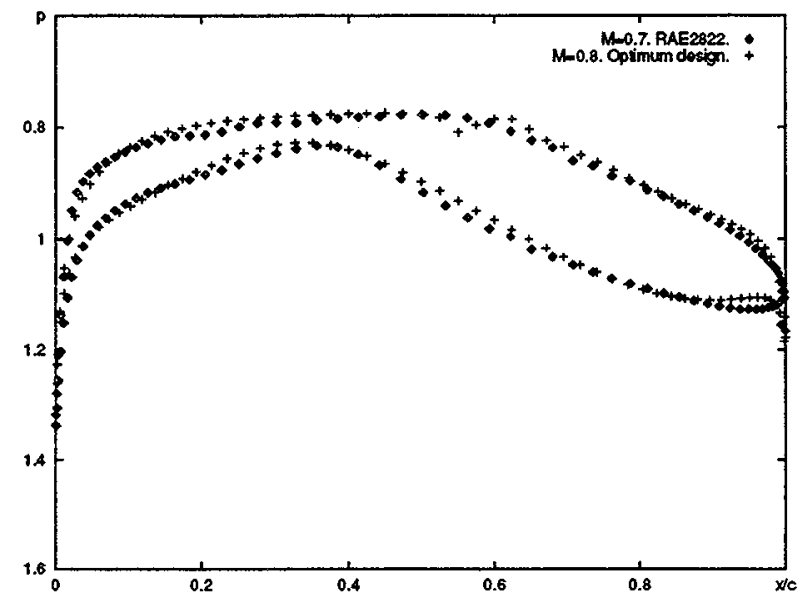

Figure 10: Pressure versus chord fraction for RAE2822 airfoil and optimal airfoil at $M_{\infty}=$ 0.80. The unperturbed flow static pressure is taken as reference.

lift using the optimization strategy that we have developed. The functional to be minimized would be a blend of drag and a quadratic penalty function for lift.

We have preferred another approach that has the advantage of controlling the target pressure distribution produced on the airfoil. In fact, pressure distribution characteristics are important for boundary layer development and transition predictions, therefore the designer needs to control directly the pressure distribution over the airfoil. Furthermore, once pressure distribution is fixed, lift, drag, and pitching moment are loosely dependent on the actual geometry of the airfoil, such that if we define some parameters that control the distribution of pressure on the airfoil and we optimize such distribution for the desired aerodynamic characteristics, we have an a priori knowledge of the pressure target.

Van Dam, van Egmond \& Slooff [26], for example, propose that the velocity distribution over the upper and lower surface be divided in three regions: stagnation, followed by a rapid 


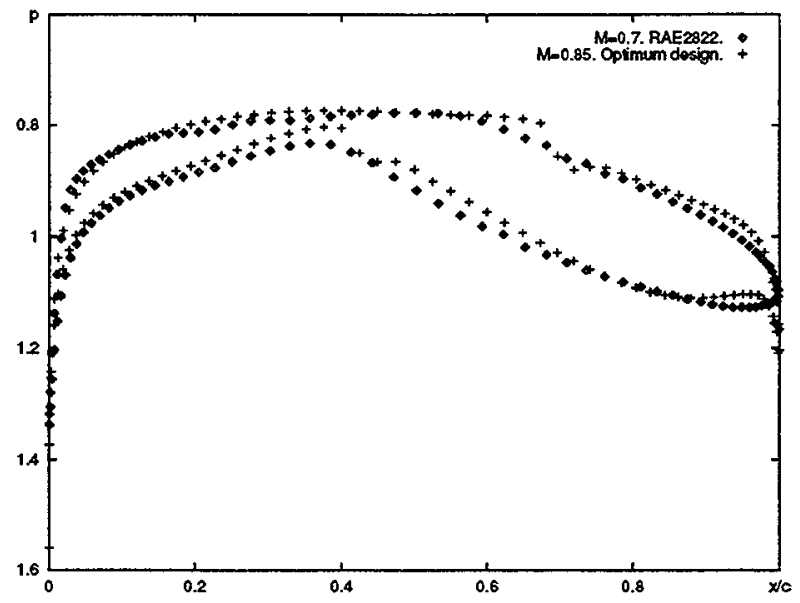

Figure 11: Pressure versus chord fraction for RAE2822 airfoil and optimal airfoil at $M_{\infty}=$ 0.85. The unperturbed flow static pressure is taken as reference.

acceleration; a region of slightly varying velocity that may be followed by a shock wave; and a pressure recovery region. These regions are defined by a number of fixed points in the pressure distribution, and the interpolation rules between these points take into account boundary layer development and separation requirements.

In this work we have limited ourself to selecting as target pressure distributions of well studied airfoils, such as a Korn airfoil, the RAE 2822, and the NACA 0012. In particular, we have studied the problem of designing a family of wing sections that produce the same surface pressure, when $M_{\infty}$ varies. The same problem was studied for the first time by Chin [6] by means of inverse design, using the full potential equations. The airfoils he obtained had the surface pressure of a Korn profile at $M_{\infty}=0.75$. These airfoils had a trailing edge angle too small for practical applications, and in some cases the upper and the lower surfaces crossed each other. The next examples show the possibility of achieving 


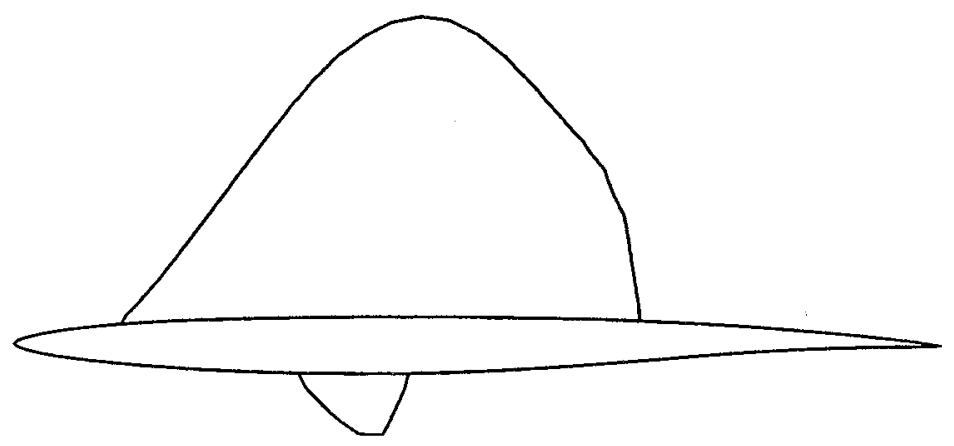

Figure 12: Supersonic bubble for AF-1 airfoil.

given aerodynamic requirements, with practical airfoils.

Using the same trailing edge of a NACA 0012 airfoil, we designed two airfoils at $M_{\infty}=$ 0.75 and $M_{\infty}=0.8$ respectively, which approximate in a least square sense the pressure distribution of a Korn airfoil at $M_{\infty}=0.7$. See figs. 5-6. In fig. 7 are presented the airfoils obtained. In fig. 8 it is shown for comparison the pressure distribution over a Korn airfoil at $M_{\infty}=0.8$.

The maximum Mach number on the airfoil $M_{\max }$, the value of the functional at the minimum $\mathcal{E}_{\min }$, the lift coefficient $C_{L}$, and wave drag $C_{D}$ for each of the unperturbed flow conditions are reported in the next table. The drag coefficient was considered zero when it was of the same order of that corresponding to numerical dissipation for subsonic test cases, i.e. $O\left(10^{-.4}\right)$. 

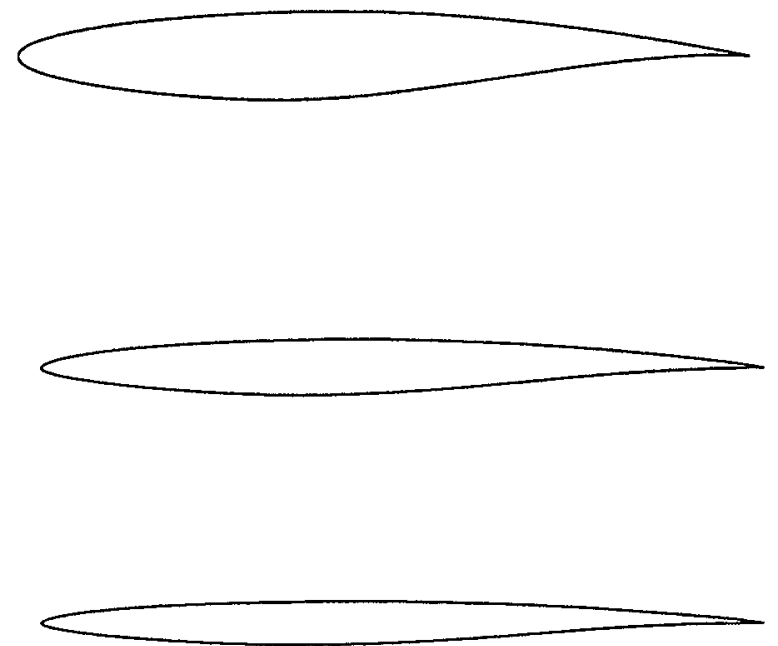

Figure 13: RAE2822 airfoil and optimal designs at $M_{\infty}=0.75, M_{\infty}=0.8$ and $M_{\infty}=0.85$.

\begin{tabular}{|l|l|l|l|l|}
\hline$M_{\infty}$ & $M_{\max }$ & $\mathcal{E}_{\min }$ & $C_{L}$ & $C_{D}$ \\
\hline 0.7 & 0.98 & & 0.44 & 0 \\
0.75 & 0.99 & $3.32 \cdot 10^{-4}$ & 0.37 & 0 \\
0.8 & 1.04 & $8.23 \cdot 10^{-4}$ & 0.31 & 0 \\
\hline
\end{tabular}

Note that the difference in lift is a measure of the first order moment of the difference $p(\Gamma)-p^{*}$

As another example of transonic design, the pressure generated over a RAE 2822 airfoil at $M_{\infty}=0.7$ was taken to be the target distribution. The trailing edge angle was required 
to be the same as that of a NACA 0012, and three flight conditions were investigated: $M_{\infty}=0.75, M_{\infty}=0.8$ and $M_{\infty}=0.85$. The pressure distributions produced on the airfoils obtained approximated to different degrees the target pressure distribution. As $M_{\infty}$ increases, the functional $\mathcal{E}$ has higher values at the minimum, i.e. for lower $M_{\infty}$ the target and the obtained pressure distribution are closer, in a least square sense. Results for each case, see also figs. $9,10,11,13$, are shown in the next table.

\begin{tabular}{|l|l|l|l|l|}
\hline$M_{\infty}$ & $M_{\max }$ & $\mathcal{E}_{\min }$ & $C_{L}$ & $C_{D}$ \\
\hline 0.7 & 0.98 & & 0.18 & 0 \\
0.75 & 1.00 & $4.03 \cdot 10^{-4}$ & 0.30 & 0 \\
0.8 & 1.02 & $5.86 \cdot 10^{-4}$ & 0.26 & 0 \\
0.85 & 1.07 & $1.03 \cdot 10^{-3}$ & 0.22 & 0 \\
\hline
\end{tabular}

The airfoil obtained for $M_{\infty}=0.85$ is shown in fig. 11, and is designated AF-1. The large supersonic bubble generated on the profile is reported in fig. 12. The AF-1 airfoil has a maximum-thickness-to-chord ratio of $6.1 \%$ and, if compared to other transonic shock-free airfoils for $M_{\infty}=0.85$, it has favorable lift characteristics. In the work of Boerstoel [4], in fact, it is conjectured that the limit maximum-thickness-to-chord ratio for a shock-free non lifting airfoil it is about $7 \%$. The nature of the recompression over the above airfoil was further investigated. The flow around the AF-1 airfoil was studied using a finer mesh with 240 tangential and 60 radial points. The entropy rise across the weak shocks on upper and lower surfaces is $O\left(10^{-3}\right)$. The lift coefficient and the drag coefficient remain unchanged compared to the coarser-grid case. 
It might be argued that the pressure distributions which were chosen for transonic design are rather peculiar: they show a typical plateau after an abrupt leading edge expansion combined with aft camber to get shockless recompression and for improving lift. For this reason, pressure distributions with other characteristics were tested, such as that of a NACA 0012 at 1 degree of incidence and $M_{\infty}=0.65$. With this distribution, we designed at $M_{\infty}=0.8$ and the results are presented in figs. 14 and 15. We have:

\begin{tabular}{|l|l|l|l|l|}
\hline$M_{\infty}$ & $M_{\max }$ & $\mathcal{E}_{\min }$ & $C_{L}$ & $C_{D}$ \\
\hline 0.65 & 0.98 & & 0.29 & 0 \\
0.8 & 1.07 & $3.79 \cdot 10^{-3}$ & 0.16 & 0 \\
\hline
\end{tabular}

Compared to the other designs at $M_{\infty}=0.8$ the approximation of the given pressure distribution is rather poor.

From the results presented it is evident that the pressure distribution that can be approximated to a higher degree is that corresponding to the RAE2822 airfoil.

\section{Conclusions}

We have designed transonic wing sections of required performance using the Euler equations. The wing sections designed have a given trailing edge angle and approximate, in a least square sense, a target pressure distribution. From a practical view point, the examples shown suggest that airfoils may be tailored for every transonic flight condition, taking into account constraints on the geometry. Furthermore we have shown that it is possible to generate profiles that approximate arbitrary pressure distributions, in particular it is pos- 


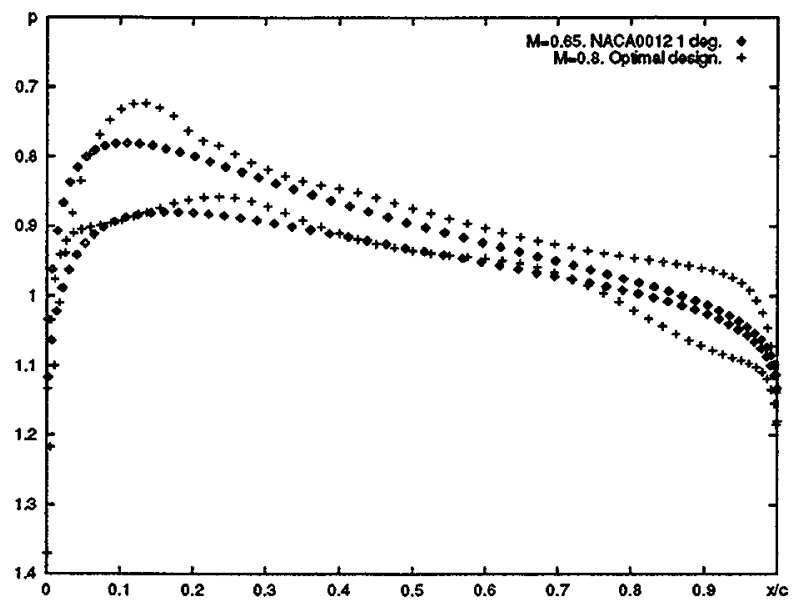

Figure 14: Pressure versus chord fraction for NACA0012 airfoil at $M_{\infty}=0.651$ degree of incidence and optimal airfoil at $M_{\infty}=0.65$.

sible to design profiles approximating a pressure distribution that satisfies requirements on pitching moment and boundary layer development.

The design was conducted by means of an optimization algorithm that uses reasonable computational resources: one full optimization costs about 5 times the cost of one analysis. The algorithm is based on the pseudo-time method and on the inverse Theodorsen transform, that allows the minimization with an increasing number of harmonics, in a natural and consistent way. With this approach the design of aerodynamic components using the Navier-Stokes equations seems to be attainable.

\section{Aknowledgments}

The first author expresses his gratitude to Professor Maurizio Pandolf for many helpful discussions about the numerical solution of the Euler equations, and to Professor Luca 


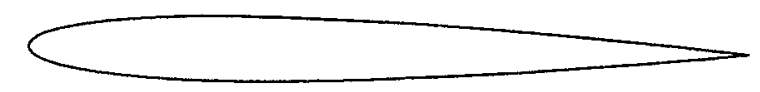

Figure 15: NACA0012 airfoil and optimal design at $M_{\infty}=0.8$.

Zannetti for his suggestions concerning the inverse Theodorsen transform.

\section{References}

[1] AGARD, Test cases for inviscid flow field methods. AGARD Report 211, p. 6-27 (1985)

[2] F. Beux and A. Dervieux, Exact-gradient shape optimization of a $2 \mathrm{D}$ Euler flow. Finite Elements in Analysis and Design, 12, pp. 281-302 (1992).

[3] F. Beux and A. Dervieux, A hierarchical approach for shape optimization. INRIA, Report 1868, Sophia Antipolis, France (February 1993).

[4] J.W. Boerstoel, Review of the application of hodograph theory to transonic aerofoil design and theoretical and experimental analysis of shock-free aerofoils. Symposium Transsonicum II, Goettingen, pp. 109-133 (1975). 
[5] H. Cabuk and V. Modi, Optimum plane diffusers in laminar flow. Journal of Fluid Mechanics, 237, pp. 373-393 (1992).

[6] W.C. Chin, Class of shock-free airfoils producing the same pressure. Journal of Aircraft, 17, pp. 286-288 (1980).

[7] R. Glowinski and O. Pironneau, On the numerical computation of the minimum-drag profile in laminar flow. Journal of Fluid Mechanics, 72, pp.385-389 (1975).

[8] A. Harten, B. Engquist, and S.R. Chakravarthy, Uniformly high order accurate essentially non-oscillatory schemes, iii. Journal of Computational Physics, 71, pp. 231-303 (1987).

[9] R. Hicks and P.A. Henne, Wing design by numerical optimization. Journal of Aircraft, 15, pp. 407-412 (1978).

[10] A. Iollo, G. Kuruvila, and S. Ta'asan, Pseudo-time method for optimal shape design using the Euler equations. AIAA Journal, 34, pp. 1807-1813 (1996).

[11] A. Iollo and M.D. Salas, Contribution to the optimal shape design of two-dimensional internal flows with embedded shocks. Journal of Computational Physics, 125, pp. 124-134 (1996).

[12] A. Iollo, M.D. Salas, and S. Ta'asan, Shape optimization governed by the Euler equations using an adjoint method. NASA CR-191555 (November 1993); see also Fourteenth 
International Conference on Numerical Methods in Fluid Dynamics, Lecture notes in Physics 453, Springer Verlag, Berlin, pp. 274-279 (1995).

[13] A. Jameson, Aerodynamic design via control theory. NASA CR-181749 (November 1988).

[14] A. Jameson and J. Reuther, Control theory based airfoil design using the Euler equations. AIAA Paper 94-4272 (1994).

[15] T.E. Labrujère and J.W. Slooff, Computational methods for the aerodynamic design of aircraft components. Annual Review of Fluid Mechanics, 25, pp. 183-214 (1993).

[16] C.S. Morawetz, On the non-existence of continuous transonic flows past profiles, i, ii, and iii. Communications in Pure and Applied Mathematics, 9, 10, and 11, pp. 45-68, pp. 107-131, and pp. 129-144 (1956, 1957 and 1958).

[17] G.Y. Nieuwland and B.M. Spee, Transonic shock-free flow, fact or fiction ? AGARD Conference Proceedings, No. 35 (1968).

[18] G.Y. Nieuwland and B.M. Spee, Transonic airfoils: Recent developments in theory, experiment and design. Annual Review of Fluid Mechanics, 5, pp. 119-150 (1973).

[19] M. Pandolf, A contribution to the numerical prediction of unsteady flows. AIAA Journal, 22, pp. 602-610 (1984).

[20] H.H. Pearcy, The aerodynamic design of section shapes for swept wings. Advances in Aeronautical Sciences, 3 (1962). 
[21] O. Pironneau, On optimum profiles in stokes flow. Journal of Fluid Mechanics, 59, pp. 117-128 (1973).

[22] O. Pironneau, On optimum design in fluid mechanics. Journal of Fluid Mechanics, 64, pp. 97-110 (1974).

[23] J. Reuther, C.P. van Dam, and R. Hicks, Subsonic and transonic low-Reynolds-number airfoils with reduced pitching moments. Journal of Aircraft, 29, pp. 297-298 (1992).

[24] J. Steinhoff and A. Jameson, Multiple solutions of the transonic potential flow equations. AIAA 5th CFD Conference, pp. 347-353 (June 1981).

[25] S. Ta'asan, Pseudo-time methods for constrained optimization problems governed by PDE. NASA CR-195081 (May 1995).

[26] R.F. van den Dam, van Egmond J.A., and J.W. Slooff, Optimization of target pressure distributions. AGARD Report 780, pp. 3-1-3-13 (1990).

[27] G. Volpe, Geometric and surface pressure restrictions in airfoil design. AGARD Report 780, pp. 4-1-4-14 (1990). 


\begin{tabular}{|c|c|c|c|}
\hline \multicolumn{3}{|c|}{ REPORT DOCUMENTATION PAGE } & $\begin{array}{l}\text { Form Approved } \\
\text { OMB No. 0704-0188 }\end{array}$ \\
\hline \multicolumn{4}{|c|}{ 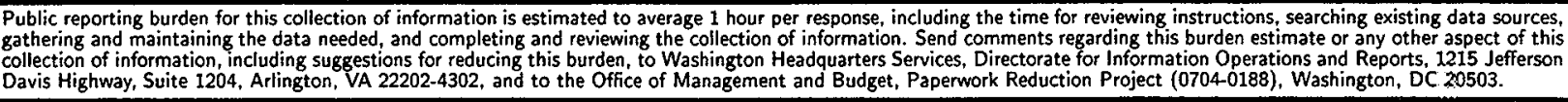 } \\
\hline 1. AGENCY USE ONLY (Leave blank) & $\begin{array}{l}\text { 2. REPORT DATE } \\
\text { December } 1996\end{array}$ & 3. RE & $\begin{array}{l}\text { ID DATES COVERED } \\
\text { rt }\end{array}$ \\
\hline \multicolumn{3}{|c|}{$\begin{array}{l}\text { 4. TITLE AND SUBTITLE } \\
\text { OPTIMUM TRANSONIC AIRFOILS } \\
\text { BASED ON THE EULER EQUATIONS }\end{array}$} & $\begin{array}{l}\text { 5. FUNDING NUMBERS } \\
\text { C NAS1-19480 } \\
\text { WU } 505-90-52-01\end{array}$ \\
\hline \multicolumn{3}{|l|}{$\begin{array}{l}\text { 6. AUTHOR(S) } \\
\text { Angelo Iollo } \\
\text { Manuel D. Salas }\end{array}$} & \\
\hline \multicolumn{3}{|c|}{$\begin{array}{l}\text { 7. PERFORMING ORGANIZATION NAME(S) AND ADDRESS(ES) } \\
\text { Institute for Computer Applications in Science and Engineering } \\
\text { Mail Stop 403, NASA Langley Research Center } \\
\text { Hampton, VA 23681-0001 }\end{array}$} & $\begin{array}{l}\text { 8. PERFORMING ORGANIZATION } \\
\text { REPORT NUMBER } \\
\text { ICASE Report No. } 96-76\end{array}$ \\
\hline \multicolumn{3}{|c|}{$\begin{array}{l}\text { 9. SPONSORING/MONITORING AGENCY NAME(S) AND ADDRESS(ES) } \\
\text { National Aeronautics and Space Administration } \\
\text { Langley Research Center } \\
\text { Hampton, VA 23681-0001 }\end{array}$} & $\begin{array}{l}\text { 10. SPONSORING/MONITORING } \\
\text { AGENCY REPORT NUMBER } \\
\text { NASA CR-201638 } \\
\text { ICASE Report No. } 96-76\end{array}$ \\
\hline \multicolumn{4}{|c|}{$\begin{array}{l}\text { 11. SUPPLEMENTARY NOTES } \\
\text { Langley Technical Monitor: Dennis M. Bushnell } \\
\text { Final Report } \\
\text { To be submitted in Computers and Fluids. }\end{array}$} \\
\hline
\end{tabular}

\begin{tabular}{l|l}
\hline 12a. DISTRIBUTION/AVAILABILITY STATEMENT & 12b. DISTRIBUTION CODE \\
Unclassified-Unlimited & \\
Subject Category 34 &
\end{tabular}

\section{ABSTRACT (Maximum 200 words)}

We solve the problem of determining airfoils that approximate, in a least square sense, given surface pressure distributions in transonic flight regimes. The flow is modeled by means of the Euler equations and the solution procedure is an adjoint-based minimization algorithm that makes use of the inverse Theodorsen transform in order to parameterize the airfoil. Fast convergence to the optimal solution is obtained by means of the pseudo-time method. Results are obtained using three different pressure distributions for several free stream conditions. The airfoils obtianed have given a trailing edge angle.

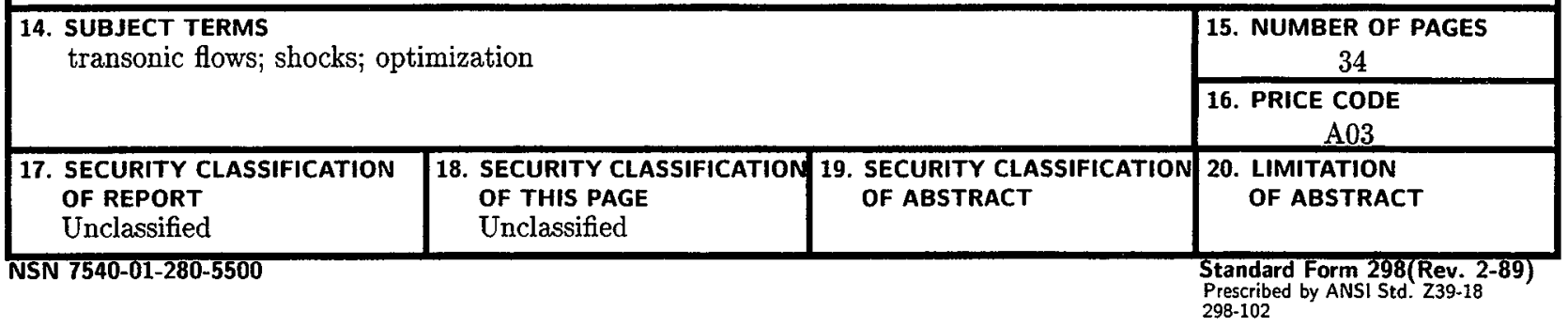

